\title{
Planar Fashionable Circuit Board Technology and Its Applications
}

\author{
Seulki Lee, Binhee Kim, and Hoi-Jun Yoo
}

\begin{abstract}
A new flexible electronics technology, named P-FCB (Planar Fashionable Circuit Board), is introduced. P-FCB is a circuit board technology implemented on the plain fabric patch for wearable electronics applications. In this paper, the manufacturing of $\mathbf{P}$ FCB, and its electrical characteristics such as sheet resistance, maximum current density, and frequency characteristics are reported. The fabrication methods and their electrical characteristics of passive devices such as resistor, capacitor, and inductor in $\mathrm{P}-\mathrm{FCB}$ are discussed. In addition, how to integrate silicon chip directly to the fabric for the flexible electronics system are described. Finally, examples of P-FCB applications will be presented.
\end{abstract}

Index Terms-Flexible circuit board, fabric, wearable computer, planar fashionable circuit board, P-FCB

\section{INTRODUCTION}

In these days, people pay attention to the wearable computer for daily applications of healthcare or entertainment. Until now, these wearable computers were implemented on the traditional PCBs or flexible circuit boards (FCBs). The PCB substrate has hardness of feeling so that it is not suitable for wearable applications, and although the FCB substrate has better flexibility than PCB, it still cannot support the fully comfortable feelings for users. To overcome this limitation, H. Kim proposed a new flexible electronics technology named P-

Manuscript received Jul. 31, 2009; revised Aug. 28, 2009.

* Dept. EE., Korea Advanced Institute of Science and Technology (KAIST), Korea

E-mail: sklee@eeinfo.kaist.ac.kr
FCB (Planar Fashionable Circuit Boards) in 2008 [1]. It is a circuit board technology implemented on the plain fabric patch for wearable electronics applications. It can support very soft and flexible feelings just same as clothes. Some characteristics of P-FCB were introduced in $[1,2]$, and this paper will provide more systematic and profound measurements on P-FCB characteristics.

The rest of the paper is organized as follows. In Section II, P-FCB will be introduced as the proposed wearable technology. The manufacturing process and parameters of P-FCB will be compared to those of the PCB. Also the electrical characteristics and mechanical characteristics of P-FCB will be explained. And the passive devices using P-FCB are shown. Section III describes the applications of P-FCB, especially focused on the daily healthcare applications. Finally, conclusions will be made in Section IV.

\section{Proposed Wearable TECHNOLOGY: P-FCB}

\section{Manufacturing process of P-FCB}

There are two kinds of printing circuit technology including thick and thin film technology [2]. In this paper, only thick film technology of screen printing process is introduced since it is more widely used due to its lower cost. The standard thick film process is comprised of printing and annealing. Some processes are correspondent with those of PCB manufacturing process. The comparison between PCB and P-FCB manufacturing process is summarized in Table 1. And the parameter comparison between PCB and P-FCB is shown in Table 
2. The all PCB parameters are based on [3], and P-FCB parameters are measured with the screen printing environment of [2]. In Table 2, it is shown that P-FCB parameters are comparable to the $\mathrm{PCB}$ parameters so that P-FCB system can replace the PCB applications.

Fig. 1 shows the DC resistance characteristics according to the various annealing temperature and time. The DC resistance is decreased when the annealing temperature is higher or the annealing time becomes longer. But higher annealing temperature or longer annealing time can makes the fabric deformed. Therefore, proper temperature and time should be determined. Fig. 1 shows the DC resistance of the silkscreen printed line with $10 \mathrm{~cm}$ length and $1 \mathrm{~mm}$ width. The conductive ink named CSP-3163 is used to print the line on the fabric [2]. Annealing time is fixed as 20 minutes in Fig. 1 (a), and the annealing temperature is fixed as $200^{\circ} \mathrm{C}$ in Fig. 1 (b). According to the measurement results in Fig. 1 (a) and (b), respectively, $300^{\circ} \mathrm{C}$ of annealing temperature and 2 hour of annealing time is shown to be most suitable since the DC resistance goes into the steadystate after that temperature and time.

After manufacturing a circuit board on fabric, several ICs should be attached to it to complete the system. The remaining process for manufacturing P-FCB system is shown in Fig. 2. First, the target IC and small metal beads are physically attached to the fabric. And thin gold wires

Table 1. Manufacturing Process Comparison.

\begin{tabular}{|c|c|c|}
\hline & PCB & P-FCB \\
\hline Step 1 & Artwork & Mask production \\
\hline Step 2 & Exposing & Screen printing \\
\hline Step 3 & Etching & Annealing \\
\hline Step 4 & Tin-Plating & Covering \\
\hline Step 5 & Drilling & - \\
\hline Step 6 & Shaping & - \\
\hline
\end{tabular}

Table 2. Manufacturing Parameter Comparison.

\begin{tabular}{|c|c|c|}
\hline & PCB & P-FCB \\
\hline Min. trace width & $0.1 \mathrm{~mm}$ & $0.1 \mathrm{~mm}$ \\
\hline \multirow{2}{*}{ Min. via size } & $0.75 \mathrm{~mm}$ (via) & \multirow{2}{*}{$2 \mathrm{~mm}$} \\
\cline { 2 - 2 } & $1.5 \mathrm{~mm}$ (barrel) & \multicolumn{2}{|c|}{} \\
\hline Min. trace spacing & $0.1 \mathrm{~mm}$ & $0.2 \mathrm{~mm}$ \\
\hline Other min. spacing & $0.1 \mathrm{~mm}$ & $0.2 \mathrm{~mm}$ \\
\hline
\end{tabular}

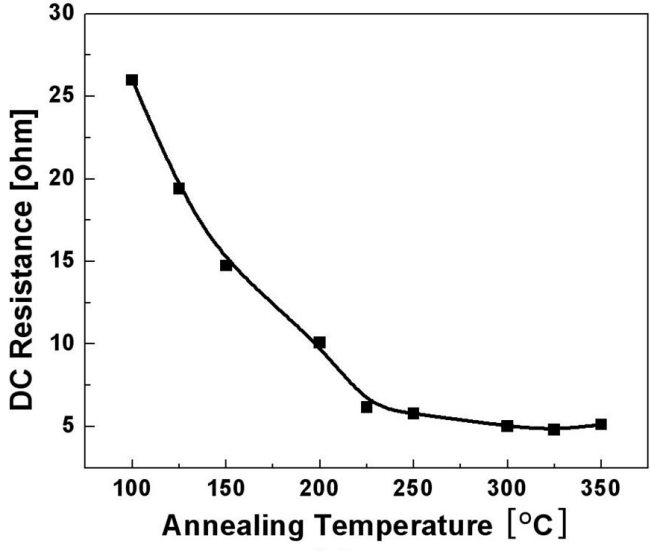

(a)

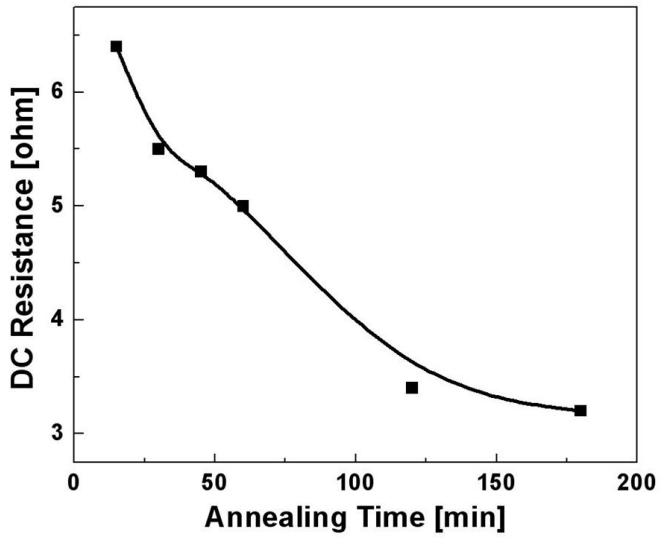

(b)

Fig. 1. Relationship between dc resistance and annealing parameters.
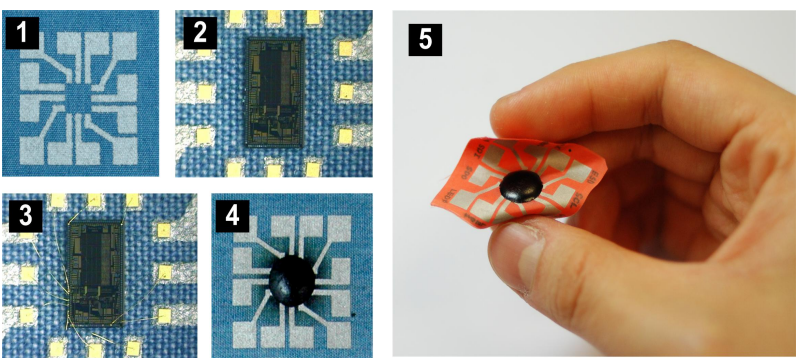

Fig. 2. P-FCB system manufacturing process.

are used to connect the pads of the IC and the circuit board. Finally, the IC and beads are molded into a package with liquid molding epoxy. It can provide highly robust protection against potential pressure on the packaged chip.

\section{Electrical characteristics of P-FCB}

To check the frequency characteristics of P-FCB, 10 $\mathrm{cm}$ long and $0.5 \mathrm{~mm}$ width wires are manufactured and tested. With Vector Network Analyzer (VNA), S21 parameters 
are measured so that the transmission efficiency is calculated. The measurement results are shown in Fig. 3. The bandwidth, which is defined as the frequency range from $0 \mathrm{~Hz}$ to below $-3 \mathrm{~dB}$ in the frequency response, is about $20 \mathrm{MHz}$. The transmission efficiency at low frequency and the $3 \mathrm{~dB}$ bandwidth is changed according to the annealing time and temperature. However, the frequency characteristics at high frequency over $3 \mathrm{~dB}$ bandwidth are similar to each other. Since lower resistance makes the transmission more efficiency, higher annealing temperature and longer annealing time is desired. And considering the deformation of fabric substrate during annealing process, it is shown that $300^{\circ} \mathrm{C}$ of annealing temperature and 2 hour of annealing time is most suitable as described in section II-A.

Fig. 4 shows the crosstalk measurement between a signal line and a neighboring line of $15 \mathrm{~cm}$ length. The signal amplitude of the aggressor is $2.5 \mathrm{~V}$ with a $6 \mathrm{~ns}$ of rising time. The space between two lines is $6 \mathrm{~mm}$. As shown in Fig. 4, the noise signal on the victim wire is $0.14 \mathrm{~V}$, which is under $5.6 \%$ of the aggression signal.

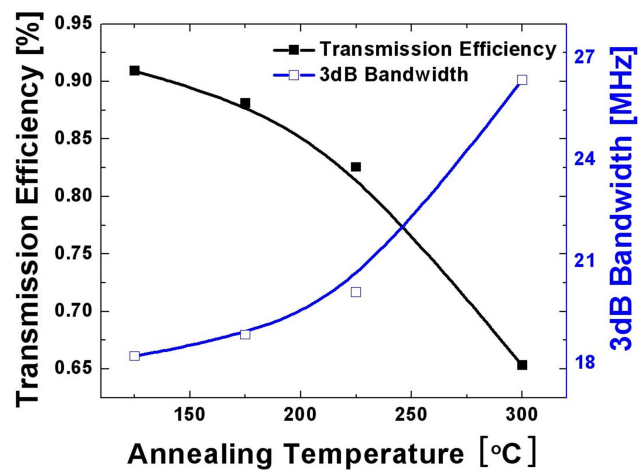

(a)

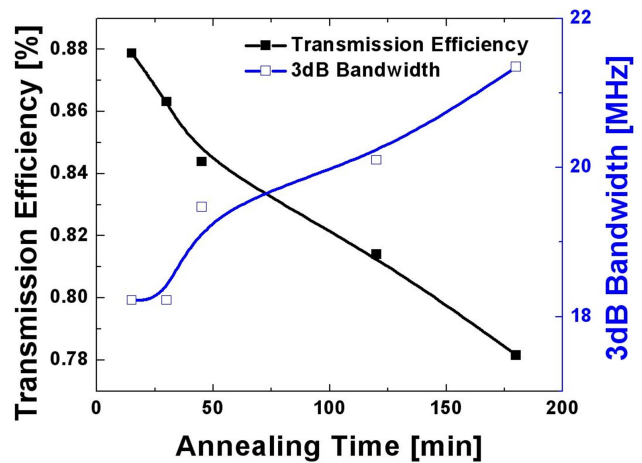

(b)

Fig. 3. Frequency characteristics of $\mathrm{P}-\mathrm{FCB}$ according to

(a) Annealing temperature with 20 minutes annealing time

(b) Annealing time with $200^{\circ} \mathrm{C}$ annealing temperature.

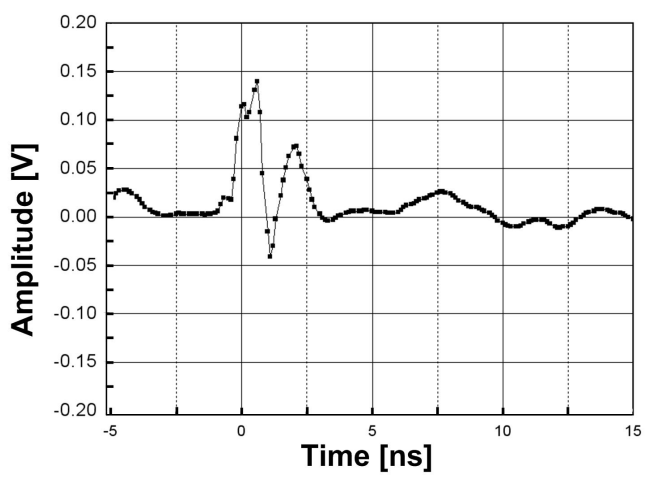

Fig. 4. Crosstalk measurement of P-FCB wires.

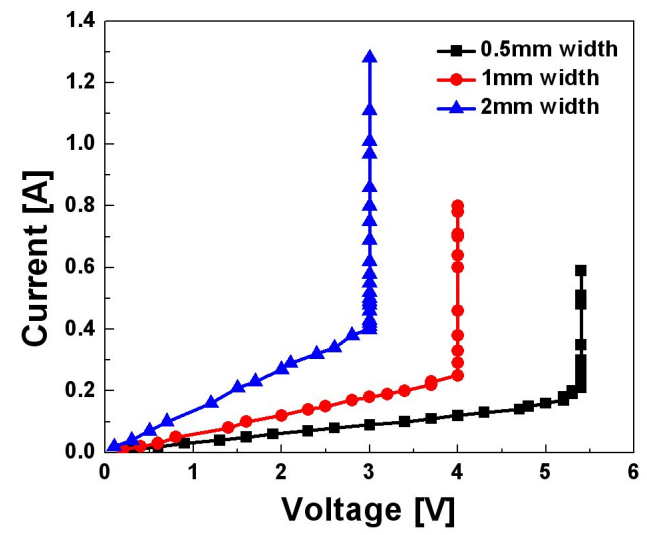

Fig. 5. Maximum current density measurement of P-FCB.

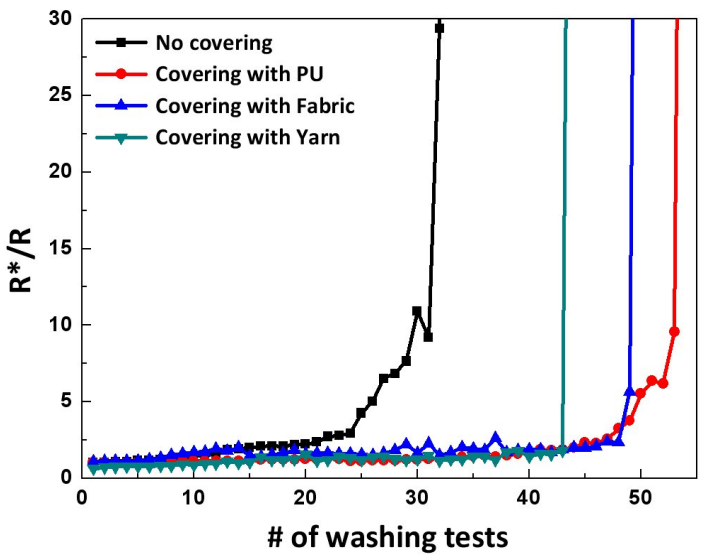

Fig. 6. Washing test results of P-FCB.

The final measurement for electrical characteristics of $\mathrm{P}-\mathrm{FCB}$ is about the maximum current density. The maximum current density is an important electrical characteristic since it determines the stability of the system against power consumption. Fig. 5 represents the measurement results on the maximum current density of P-FCB wires. It differs from the wire length and width because a wire resistance is changed according to them. 


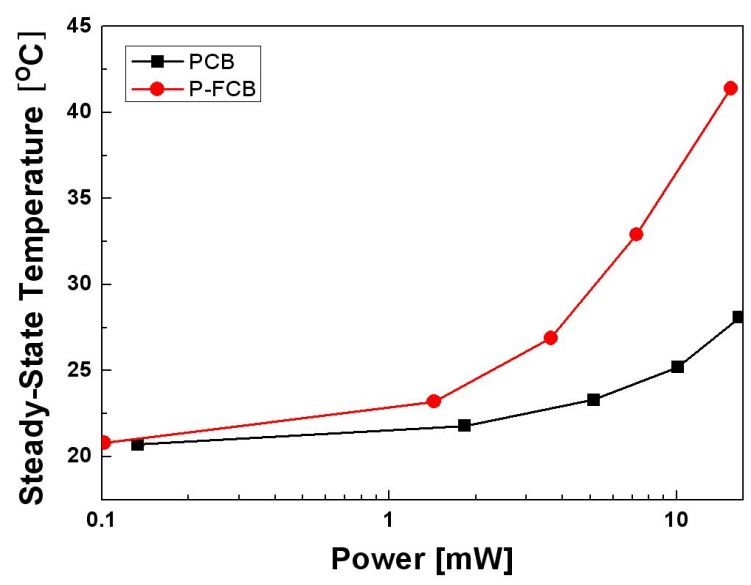

Fig. 7. Comparison of heat dissipation between P-FCB and PCB.

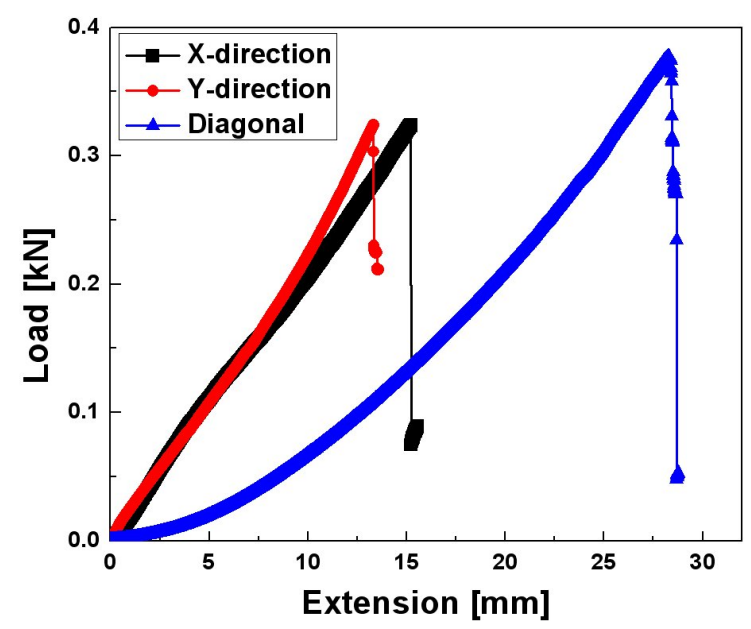

Fig. 8. Durability to tear of P-FCB system.

Table 3. Fabric Characteristics.

\begin{tabular}{|c|c|}
\hline Characteristics & Value \\
\hline Yarn fineness $($ tex $)$ & 4.4 \\
\hline Fabric density $\left(\mathrm{dm}^{-1}\right)$ & 500 \\
\hline Volume resistivity & - \\
\hline Dielectric permittivity $\left(\varepsilon_{\mathrm{r}}\right)$ & 4.0 \\
\hline Dielectric strength $(\mathrm{kV} / \mathrm{m})$ & - \\
\hline
\end{tabular}

As described before, annealing temperature and annealing time affect the wire resistance so that it also affects the maximum current density. In this measurement, $300^{\circ} \mathrm{C}$ and 2 hour annealing environment is used. As shown in Fig. 5, 0.17 A, 0.2 A, and 0.4 A of current will be stood for $0.5 \mathrm{~mm}, 1 \mathrm{~mm}$, and $2 \mathrm{~mm}$ of the wire width, respectively. The linear region at low voltage of the graph is caused by wire resistance.

\section{Mechanical characteristics of P-FCB}

The durability to washing of P-FCB system is shown in Fig. 6. Three covering methods are introduced, and non-covered P-FCB is also measured as a reference. As shown in Fig. 6, all of covering methods show better durability to washing than P-FCB itself without covering. However, even for non-covered P-FCB, it passes over 30 times of washing tests. This mechanical durability to washing is enough for wearable system embedded in our everyday dress. So we can add the covering process only when the specially enhanced durability is required.

Fig.7 shows the comparison of heat dissipation between P-FCB and PCB. The measurement result represents that $\mathrm{P}-\mathrm{FCB}$ has worse characteristics for heat dissipation than PCB above $1 \mathrm{~mW}$ of power. This result becomes the important indicator of P-FCB system design. To make P-FCB system operate safely, it should consume power less than $1 \mathrm{~mW}$. Within this power range, the operating

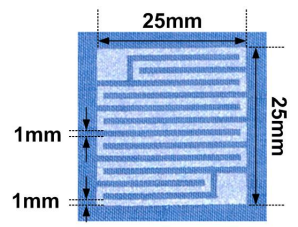

(a) Resistor

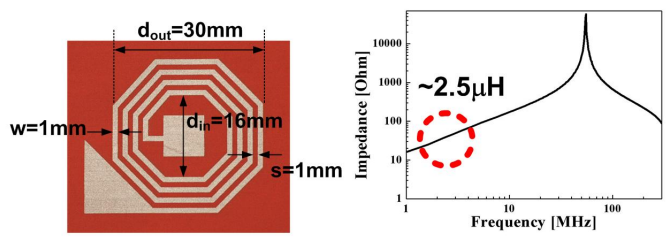

(b) Inductor
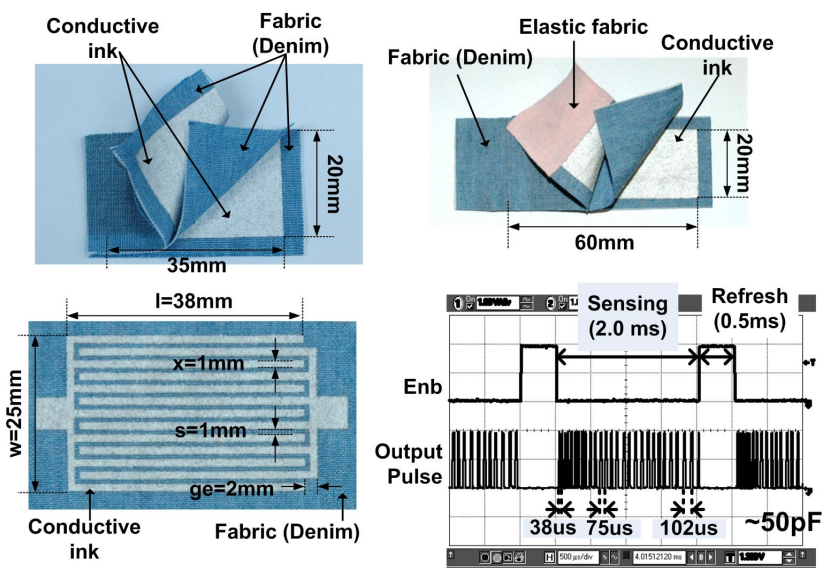

(c) Three types of capacitor

Fig. 9. Passive devices using P-FCB. 
Table 4. CSP-3225 Characteristics.

\begin{tabular}{|c|c|}
\hline Characteristics & Value \\
\hline Solid Content & 41.79 \\
\hline Viscosity & 190 \\
\hline Fineness of Grind (FoG) & 5 \\
\hline Specific Gravity & 1.27 \\
\hline Adhesive Strength & $100 / 100$ \\
\hline Resistivity & 3.28 \\
\hline Crease Test & 4.80 \\
\hline Pencil Hardness & 3 \\
\hline
\end{tabular}

temperature of P-FCB system does not increase over 5\% of PCB system. Otherwise, P-FCB system may suffer from excessive heat dissipation which induces high operating temperature and fault operation.

The durability to tear of P-FCB system is measured as shown in Fig. 8. The measurement in this study is performed by using the fabric made with polyester (PES). And it is revealed that the durability of fabric is weaker than that of conductive ink on it so the durability to tear of P-FCB system mainly depends on the type of fabric. In our system, almost $300 \mathrm{~N}$ of $\mathrm{x}$ - or $\mathrm{y}$-directional force can be withstood without any defect. And it can endure $380 \mathrm{~N}$ of force with $28 \mathrm{~mm}$ extension in diagonal direction. Note that it is the result of a specific fabric made with PES whose characteristics are represented in Table 3, so it can be changed according to the fabric substrate in P-FCB system. And by considering our everyday dress, the durability to tear is strong enough.

\section{Passive devices using P-FCB}

In the conventional PCB system, surface-mounted type of passive devices such as resistor, capacitor, and inductor are used. However, in the proposed P-FCB system, these passive devices can be implemented using same fabrication process as a circuit board. Fig. 9 shows the examples of passive devices using P-FCB. The sheet resistance of the resistor is $45.8 \Omega / \square$ when 3 times of printing with $300^{\circ} \mathrm{C}$ and 2 hour annealing is performed. To make the resistor, new conductive ink named CSP3225 is employed which has larger resistance and equal flexibility compared with CSP-3163. The characteristics of CSP-3225 are summarized in Table 4.

Three types of capacitor and one type of inductor is also proposed in Fig. 9. The capacitance range is between $1 \mathrm{pF}$ and $100 \mathrm{pF}$ [2], and the inductance range is between $100 \mathrm{nH}$ and few $\mu \mathrm{H}$ [2], [5]. Using a capacitor sensing

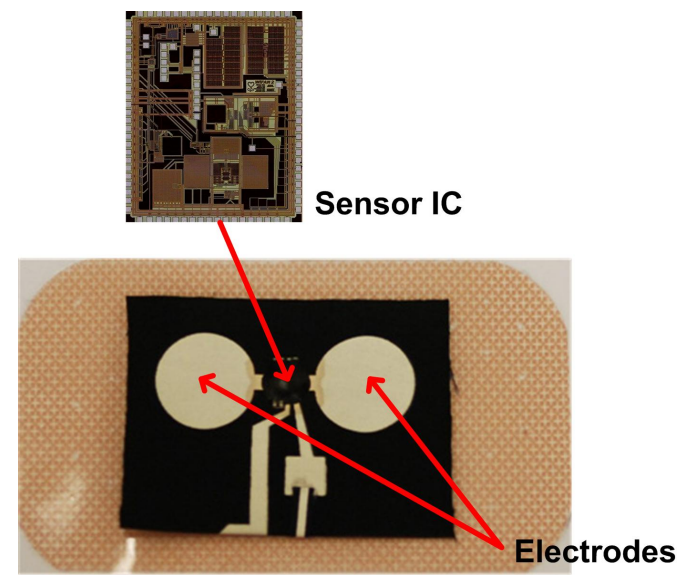

Fig. 10. ECG sensor node using P-FCB [4].

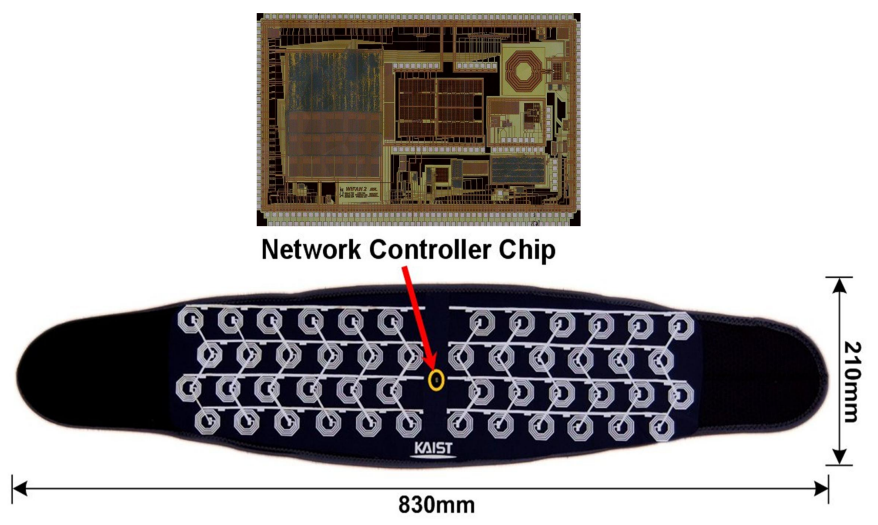

Fig. 11. Inductor channel array implemented on the chest band [4].

system on P-FCB, the capacitance can be calculated [1]. As shown in Fig. 9 (c), the number of output pulses from the IC is increased by larger capacitance. So we can count the number of output pulses to see the target capacitance. Also we can estimate the inductance by using LC tank and phase difference [6]. To make the product of inductance and known capacitance constant, we can see the inductance variation. If the carrier frequency of the clock signal is also known, then we can calculate the inductance exactly. By using these passive devices, P-FCB system can increase the wearability compared to PCB system.

\section{Application OF P-FCB}

Basically, all systems which can be implemented with PCB are potential applications of P-FCB. Among them, the most appropriate application is healthcare system if 
we pay attention to the wearable characteristics of PFCB. Several healthcare systems using P-FCB were introduced [1], [2], and [4], and all of them are more suitable compared with previous PCB systems. In this section, some examples of P-FCB applications are presented.

Fig. 10 shows the ECG sensor node which is implemented with P-FCB. It is already shown that these sensor nodes can be implemented as a band-aid type [4]. The electrodes are placed on the fabric, and sensor AFE and transceiver circuits are integrated into the SoC of PFCB. So the fabric patch is in charge of both sensing and data transmission. To reduce the thermal and $1 / \mathrm{f}$ noise from the P-FCB electrode, sensor AFE circuits adopts the chopping techniques as in [7]. The data transmission in [4] is performed by inductive coupling. The communication channel is a pair of inductor which is described in section II-D. And the inductor channel array used in [4] is shown in Fig. 11. It is a kind of chest band what people sometimes use in daily life. As shown in Fig.11, P-FCB also has high potential in the channel of nearfield communication for wearable computer. Since nearfield communication is suitable for communication in the wearable computer field due to its low power consumption characteristic $[5,6,8]$, P-FCB system can be widely used in that area. Although the flexibility of PFCB becomes a problem in this application since the inductance can be changed during communication, the compensation technique is proposed so that it makes the communication more reliable [6].

\section{CONCLUSIONS}

A new flexible electronics technology named P-FCB is introduced. Fabrication process and comparison with PCB are represented. And some electrical and mechanical characteristics are measured to see the suitability of PFCB to wearable computer applications. The measurement results reveal that P-FCB can be a good circuit board as previous PCB with improved flexibility. Finally, the applications of P-FCB are shown that P-FCB has a great potential to the healthcare system and wearable system.

\section{ACKNOWLEDGMENT}

This work was supported by the IT R\&D program of the Korean MKE and KEIT(2008-F-048, Wearable Personal Companion for u-computing collaboration).

\section{REFERENCES}

[1] Hyejung Kim, Yongsang Kim, Young-Se Kwon, and Hoi-Jun Yoo, "A 1.12mW Continuous Healthcare Monitor Chip Integrated on a Planar Fashionable Circuit Board," ISSCC Digest of Technical Papers, pp.150-603, Feb., 2008.

[2] Yongsang Kim, Hyejung Kim, and Hoi-Jun Yoo, "Electrical Characterization of Printed Circuits on Fabric," IEEE Transactions on Advanced Packaging (Submitted).

[3] Jon Varteresian, "Fabricating Printed Circuit Boards," Newnes, 2002.

[4] Jerald Yoo, Long Yan, Seulki Lee, Yongsang Kim, Hyejung Kim, Binhee Kim, and Hoi-Jun Yoo, "A $5.2 \mathrm{~mW}$ Self-Configured Wearable Body Sensor Network Controller and a $12 \mu \mathrm{W} 54.9 \%$ Efficiency Wirelessly Powered Sensor for Continuous Health Monitoring System," ISSCC Digest of Technical Papers, pp.290-291, Feb., 2009.

[5] Jerald Yoo, Seulki Lee, and Hoi-Jun Yoo, "A $1.12 \mathrm{pJ} / \mathrm{b}$ Resonance Compensated Inductive Transceiver with a Fault-Tolerant Network Controller for Wearable Body Sensor Network," IEEE Asian Solid-State Circuit Conference, pp.313-316, Nov., 2008.

[6] Seulki Lee, Jerald Yoo, Hyejung Kim, and Hoi-Jun Yoo, "A Dynamic Real-time Capacitor Compensated Inductive Coupling Transceiver for Wearable Body Sensor Network," IEEE Symposium on VLSI Circuits, pp.42-43, Jun., 2009.

[7] Long Yan, Namjun Cho, Jerald Yoo, Binhee Kim, and Hoi-Jun Yoo, "A Two-Electrode 2.88nJ/Conversion Biopotential Acquisition System for Portable Healthcare Device," IEEE Asian Solid-State Circuits Conference, pp.329-332, Nov., 2008.

[8] Esko Strömmer, Jouni Kaartinen, Juha Pärkkä, Artoo Ylisaukko-oja, and Ilkka Korhonen, "Application of Near Field Communication for Health 
Monitoring in Daily Life," Proceedings of the $28^{\text {th }}$ IEEE EMBS Annual International Conference, pp. 3246-3249, Sep., 2006.

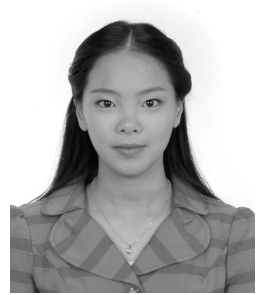

Seulki Lee (S'07) received the B.S. and M.S. degree from Korea Advanced Institute of Science and Technology (KAIST), Daejeon, Korea, in 2007 and 2009, respectively, where she is currently working toward the $\mathrm{Ph} . \mathrm{D}$. degree, all in electronic engineering.

Her current research interests include the inductive coupling transceiver design and near-field communication for wearable computing applications.

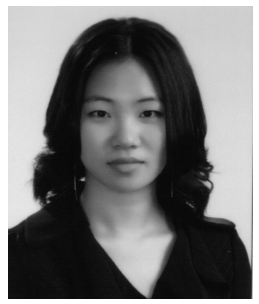

Binhee Kim (S'08) received the B.S. degree in electrical engineering from the Korea Advanced Institute of Science and Technology (KAIST), Daejeon, Korea, in 2008.

She is currently working toward the master degree in the same department at KAIST.

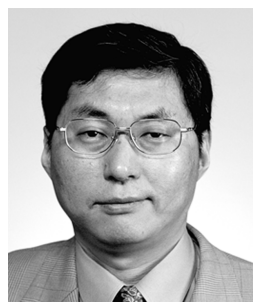

Hoi-Jun Yoo (M'95-SM'04-F'08) graduated from the Electronic Department of Seoul National University, Seoul, Korea, in 1983 and received the M.S. and Ph.D. degrees in electrical engineering from the Korea Advanced Institute of Science and Technology (KAIST), Daejeon, in 1985 and 1988, respectively. His Ph.D. work concerned the fabrication process for GaAs vertical optoelectronic integrated circuits.
From 1988 to 1990, he was with Bell Communications Research, Red Bank, NJ, where he invented the twodimensional phased-locked VCSEL array, the frontsurface-emitting laser, and the high-speed lateral HBT. In 1991, he became Manager of a DRAM design group at Hyundai Electronics and designed a family of from fast-1M DRAMs and 256M synchronous DRAMs. In 1998 he joined the faculty of the Department of Electrical Engineering at KAIST and now is a full professor. From 2001 to 2005, he was the director of System Integration and IP Authoring Research Center (SIPAC), funded by Korean government to promote worldwide IP authoring and its $\mathrm{SoC}$ application. From 2003 to 2005, he was the full time Advisor to Minister of Korea Ministry of Information and Communication and National Project Manager for SoC and Computer. In 2007, he founded SDIA (System Design Innovation \& Application Research Center) at KAIST to research and develops SoCs for intelligent robots, wearable computers and biosystems. His current interests are high-speed and low-power Network on Chips, 3D graphics, Body Area Networks, biomedical devices and circuits, and memory circuits and systems. He is the author of the books DRAM Design (Seoul, Korea: Hongleung, 1996; in Korean), High Performance DRAM (Seoul, Korea: Sigma, 1999; in Korean), and chapters of Networks on Chips (New York, Morgan Kaufmann, 2006).

Dr. Yoo received the Electronic Industrial Association of Korea Award for his contribution to DRAM technology the 1994, Hynix Development Award in 1995, the Korea Semiconductor Industry Association Award in 2002, Best Research of KAIST Award in 2007, Design Award of 2001 ASP-DAC, and Outstanding Design Awards 2005, 2006, 2007 A-SSCC. He is a member of the executive committee of ISSCC, Symposium on VLSI, and A-SSCC. He is the TPC chair of the A-SSCC 2008. 